\title{
A Non-immunogenic Bivalent D-Protein Potently Inhibits Retinal Vascularization and Tumor Growth
}

Paul S. Marinec ${ }^{1,{ }^{*}}$, Kyle E. Landgraf ${ }^{1, *}$, Maruti Uppalapati ${ }^{1,2, \dagger}$, Gang Chen $^{1,2}$, Daniel Xie ${ }^{3}$, Qiyang Jiang ${ }^{4}$, Yanlong Zhao ${ }^{4}$, Annalise Petriello ${ }^{5}$, Kurt Deshayes ${ }^{1, \$}$, Stephen B.H. Kent ${ }^{1}$, Dana Ault-Riche ${ }^{1, \S}$ and Sachdev S. Sidhu ${ }^{1,2, \S}$

${ }^{1}$ Reflexion Pharmaceuticals, Incline Village, NV 89451.

${ }^{2}$ Department of Molecular Genetics, The Donnelly Centre, University of Toronto, Toronto, ON, Canada, M5S 3E1.

${ }^{3}$ Chinese Peptide Company, Hangzhou Economic and Technical Development Zone, China 310018.

${ }^{4}$ Viva Biotech, Zhangjiang High-Tech Park, Shanghai, China 201203.

5 iQ Biosciences, Berkeley, CA 94710

*These authors contributed equally to this work

${ }^{\S}$ Correspondence to: aultriche@ reflexionpharma.com, sidhu@ reflexionpharma.com

${ }^{\dagger}$ Current address: Department of Pathology and Laboratory Medicine, University of

Saskatchewan, Saskatoon, SK, Canada.

${ }^{\star}$ Current address: Chemical Biology Consultants, San Francisco, CA 94133 


\section{Supporting Information:}

Methods

Figures S1-S6

Tables S1-S4

\section{Methods}

Chemical Synthesis Reagents

Fmoc-D-amino acids were purchased from Chengdu Zhengyuan Company, Ltd and Chengdu Chengnuo New-Tech Company, Ltd. Fmoc-D-Ile-OH was purchased from Chemimpex International, Inc. Fmoc-D-propargylglycine (Fmoc-D-Pra-OH) was purchased from Haiyu Biochem. MBHA Resin was purchased from Sunresin New Materials Co. Ltd., Xian. Rink Amide linker was purchased from Chengdu Tachem Company, Ltd. Chloro-(2-Cl)trityl-resin was purchased from Tianjin Nankai Hecheng Science and Technology Company, Ltd. Fmoc-NH2(PEG)n-COOH and other PEG linkers were purchased from Biomatrik Inc. 2Azidoacetic acid was purchased from Amatek Scientific Company Ltd. Sodium ascorbate was purchased from TCI (Shanghai) Ltd. Copper sulfate pentahydrate $\left(\mathrm{CuSO}_{4} \cdot 5 \mathrm{H}_{2} \mathrm{O}\right)$ was purchased from Energy Chemical.

\section{D-VEGF-A synthesis and refolding}

The D-VEGF-A polypeptide chain (COOH acid, residues 8-109 (1)) was chemically synthesized using solid phase peptide synthesis (SPPS) and native chemical ligation, and folded to form the protein covalent homodimer, using methods adapted from our previous work (2)

adapted to Fmoc chemistry SPPS (3). Individual peptide fragments corresponding to 1: Gly ${ }^{1}$-to- 
D-Tyr ${ }^{18}, 2$ : D-Cys ${ }^{19}$-to- D-Arg ${ }^{49}$, 3: D-Cys ${ }^{50}$-to- D-Asp ${ }^{102}$, were synthesized using standard Fmoc chemistry protocols for stepwise SPPS. Fragments 1 and $\mathbf{2}$ were synthesized on $\mathrm{NH}_{2} \mathrm{NH}-(2-$ Cl)trityl-resin and fragment 3 was synthesized from pre-loaded Wang Resin. Briefly, preloaded Fmoc-aminoacyl-Wang Resin was initially swelled with DMF $(10 \mathrm{~mL} / \mathrm{g})$ for 1 hour, then treated with $20 \%$ piperidine/DMF (30 min) to remove the Fmoc group and washed again with DMF (5 times). Fmoc-D-amino acid residues were coupled by addition of a pre-activated solution of 3 equivalents each of protected amino acid (0.4 M in DMF), diisopropylcarbodiimide (DIC), and hydroxybenzotriazole (HOBt) to the resin. After 1-2 h, the ninhydrin test showed the reaction was completed and the resin was washed with DMF (3 times). To remove the Fmoc group, piperidine (20\% in DMF) was added to the resin for 30 min. After removal of the final Fmoc group, the resin was rinsed with DMF (3 times) and $\mathrm{MeOH}$ (2 times), dried under vacuum, then taken up in $85 \%$ TFA, $5 \%$ thioanisole, $5 \%$ EDT, $2.5 \%$ phenol and $2.5 \%$ water for cleavage from the resin and concomitant removal of side chain protecting groups. After $2 \mathrm{~h}$, the resin was washed with TFA and the eluted peptide was concentrated by bubbling nitrogen gas. The crude peptides were precipitated with cold ether, pelleted by centrifugation, and washed with cold ether 2 times before drying under vacuum. The peptide residue was dissolved in water, purified by preparative reverse phase HPLC and analyzed by HPLC and MS.

Ligations between D-peptide-hydrazide fragments and D-Cys-peptide fragments were performed as follows: D-peptide-hydrazide was dissolved in Buffer A (0.2M sodium phosphate containing $6 \mathrm{M} \mathrm{GnHCl}, \mathrm{pH} 3.0$ ), cooled to $-15^{\circ} \mathrm{C}$ in an ice-salt bath, and gently stirred by magnetic stirrer. $\mathrm{NaNO}_{2}$ (7 equivalents) was added and the solution stirred for 20 min to oxidize the D-peptide-hydrazide to the D-peptide-azide. A solution of 4-mercaptophenylacetic acid (MPAA) (50 eq) dissolved in Buffer B (0.2M sodium phosphate containing $6 \mathrm{M} \mathrm{GnHCl,} \mathrm{pH} \mathrm{7.0)}$ 
was quickly added to the solution containing the newly-formed D-Peptide-azide (equal volume) to eliminate excess $\mathrm{NaNO}_{2}$ and to convert the peptide-azide to the peptide-MPAA thioester. Then a solution of D-Cys-peptide in Buffer B (equal volume) was added to the solution containing the newly formed peptide-MPAA thioester. The reaction mixture was adjusted to $\mathrm{pH}$ 7 with $\mathrm{NaOH}$ to initiate overnight native chemical ligation. Reaction progress was monitored by analytic RP-HPLC until completion, then treated with TCEP (25 eq) before HPLC purification.

Purification of the ligated polypeptide product was performed on a CXTHLC6000/Hanbon NU3000 prep system on Phenomenex C18/YMC C4 silica with columns of dimension 21.2×250 $\mathrm{mm} / 20.0 \times 250 \mathrm{~mm}$. Crude product was loaded onto the prep column and eluted at a flow rate of $5 \mathrm{~mL}$ per minute with a shallow gradient of increasing concentrations of solvent $\mathrm{B}(0.1 \%$ TFA in $80 \%$ acetonitrile $)$ in solvent $\mathrm{A}(0.1 \%$ TFA in water). Fractions containing the purified target polypeptide were identified by analytical LC-MS, combined, and lyophilized.

Final linear D-VEGF-A 102 residue polypeptide chain was folded at $\mathrm{pH} 8.4$ in aqueous $\mathrm{Gu} \cdot \mathrm{HCl}(0.15 \mathrm{M})$ containing a glutathione-reduced $(2 \mathrm{mM}) /$ glutathione-oxidized $(0.4 \mathrm{mM})$ redox couple and stirred for 5 days to reach completion (2). Folded D-VEGF-A covalent homodimer was purified by RP-HPLC.

\section{Phage display libraries and panning}

Naïve GA- and Z-domain scaffold libraries were constructed as fusions to the N-terminal gene 8 major coat protein by previously described methods (4). Randomization of desired library positions (Fig. S1) was performed using Kunkel mutagenesis (5) with trinucleotide oligos allowing incorporation of all-natural amino acids except cysteine. The resulting libraries contained $>10^{10}$ unique members. For affinity maturation libraries, Kunkel mutagenesis was 
performed on RFX-V1 or RFX-V2 parent sequences using targeted NNC or soft-randomization oligos, respectively. Positions targeted for affinity maturation are highlighted in Figure S1.

All phage selections were executed according to previously established protocols (2). Briefly, selections with the peptide libraries were performed using biotinylated D-VEGF captured with streptavidin-coated magnetic beads (Promega). Initially, three rounds of selection were completed with decreasing amounts of D-VEGF $(2.0 \mu \mathrm{M}, 1.0 \mu \mathrm{M}$, and $0.5 \mu \mathrm{M})$. The phage pools were then transferred to a $\mathrm{N}$-terminal gene 3 minor coat protein display vector and subjected to an additional three rounds of panning with decreasing amounts of D-VEGF (200 $\mathrm{nM}, 100 \mathrm{nM}$, and $50 \mathrm{nM}$ ) and increased wash times. Individual phage clones were then sent in for sequencing analysis.

\section{Synthesis of D-protein binders}

The polypeptide chains of the D-proteins RFX-V1, -V1a, -V2, and -V2a (Fig. S1) were prepared manually by Fmoc chemistry stepwise SPPS on Rink Amide MBHA Resin. Side-chain protection for amino acids was as follows: D-Arg(Pbf), D-Asp(OtBu), D-Glu(OtBu), DAsn(Trt), D-Gln(Trt), D-Ser(tBu), D-Thr(tBu), D-Tyr(tBu), D-His(Trt), D-Lys(Boc), D$\operatorname{Trp}(\mathrm{Boc})$. After chain assembly of the D-polypeptides was complete and the final Fmoc group removed, the resulting D-peptides had their side-chains deprotected and were simultaneously cleaved from the resin support by treatment with TFA containing $2.5 \%$ triisopropylsilane and $2.5 \% \mathrm{H}_{2} \mathrm{O}$ for $2.5 \mathrm{~h}$ at room temperature. Crude D-polypeptide products were recovered from resin by filtration, precipitated, and triturated with chilled diethyl ether then dried under vacuum. To form the single disulfide bond, crude RFX-V1a was oxidized using I2. Briefly, crude peptide was dissolved in $30 \%$ acetonitrile and acidified with acetic acid. An I2/MeOH solution was 
added dropwise until the mixture was pale yellow then aqueous sodium ascorbate was added dropwise to quench excess $\mathrm{I}_{2}$. D-polypeptide chains folded spontaneously upon dissolution in appropriate buffer to yield the functional D-protein binder molecules.

Synthesis of the D-protein Heterodimer RFX-VIa2a

Step 1: Preparation of Azido-PEG3-D-RFX-Vla Resin. Fmoc-aminoacyl-Rink Amide MBHA Resin was swelled in DMF (10-15 mL/g resin) for 1 hour. The suspension was filtered, exchanged into DMF containing $20 \%$ piperidine, and kept at room temperature for $0.5 \mathrm{hr}$ under continuous nitrogen gas perfusion. The resin was then washed 5 times with DMF. Fmoc-Damino acid-OH, DIC, HOBt and DMF were added to the resin. The suspension was kept at room temperature for $1 \mathrm{hr}$ while a stream of nitrogen was bubbled through it. The ninhydrin test was used to monitor the coupling reaction until completion. The remaining D-amino acids corresponding to the affinity matured D-protein RFX-V1a were sequentially coupled to the peptidyl resin by removal of the Fmoc group, washing, and addition of the appropriate activated Fmoc amino acid. Amino acid residue D-Lys ${ }^{19}$ was introduced with orthogonal side chain protection as Fmoc-D-Lys(Dde)-OH. After assembly of the amino acid sequence of the protected RFX-V1a polypeptide chain was complete, the Dde group was removed by treatment with $2 \%$ hydrazine in DMF, then azido-PEG3-COOH was coupled to the primary amine of Lys ${ }^{19}$ and the N-terminal Fmoc group was removed by treatment with DMF containing $20 \%$ piperidine. The peptidyl-resin was washed with DMF (5 times), MeOH (2 times), DCM (2 times) and MeOH (2 times), then dried under vacuum overnight. 
Step 2: Cleavage and Deprotection of Azido-PEG3-D-RFX-Vla-Resin. Cleavage solution (TFA/Thioanisole/EDT/Phenol/ $\mathrm{H}_{2} \mathrm{O}=87.5 / 5 / 2.5 / 2.5 / 2.5 \mathrm{v} / \mathrm{v}, 10 \mathrm{~mL} / \mathrm{g}$ peptide Resin) was added to the dried Azido-PEG3-D-RFX-V1a-resin. The suspension was shaken for $3 \mathrm{~h}$ and was filtered and the filtrate collected. Cold ether was added to the filtrate to precipitate the peptide which was recovered by centrifugation. The white precipitate was washed with ether twice, then dried under vacuum overnight to give crude Azido-PEG3-D-RFX-V1a as a white solid.

Step 3: Oxidation and Purification. Crude Azido-PEG3-D-RFX-V1a was oxidized using I2. Briefly, peptide $(23.5 \mathrm{mg}$ ) was dissolved in $11 \mathrm{~mL}$ of $30 \% \mathrm{ACN}$ and mixed with $330 \mu \mathrm{L}$ of $\mathrm{CH}_{3} \mathrm{COOH}$. An $\mathrm{I}_{2} / \mathrm{MeOH}$ solution was added dropwise until the mixture was pale yellow then aqueous sodium ascorbate was added dropwise to quench excess $\mathrm{I}_{2}$. Purification of oxidized Azido-PEG3-D-RFX-V1a was performed on a CXTH LC6000/Hanbon NU3000 prep system on Phenomenex C18 silica with columns of dimension $21.2 \times 250 \mathrm{~mm}$. Crude peptides were loaded onto the prep column and eluted at a flow rate of $5 \mathrm{~mL} / \mathrm{min}$ with a shallow gradient of increasing concentrations of solvent B $(0.1 \%$ TFA in $80 \%$ acetonitrile in water) in solvent A $(0.1 \%$ TFA in water). Fractions containing the pure target peptide were identified by analytical LC-MS, and were combined and lyophilized to give purified Azido-PEG3-D-RFX-V1a for subsequent click reaction with (Alkynyl-PEG2)-D-RFX-V2a.

Step 4: Preparation of Alkynyl-PEG2-D-RFX-V2a resin. Fmoc-aminoacyl-Rink Amide MBHA Resin was swelled in DMF (10-15 mL/g resin) for 1 hour. The suspension was filtered, exchanged into DMF containing 20\% piperidine, and kept at room temperature for $0.5 \mathrm{hr}$ under 
continuous nitrogen gas perfusion. The resin was then washed 5 times with DMF. Fmoc-Damino acid-OH, DIC, HOBt and DMF were added to the resin. The suspension was kept at room temperature for $1 \mathrm{hr}$ while a stream of nitrogen was bubbled through it. The ninhydrin test was used to monitor the coupling reaction until completion. The remaining D-amino acids corresponding to the affinity matured D-protein RFX-V2a polypeptide chain were coupled to the peptide-resin by removal of the Fmoc group and addition of the appropriate activated Fmoc amino acid, sequentially, in order. Amino acid residue D-Lys ${ }^{7}$ was introduced with orthogonal side chain protection as Fmoc-D-Lys(Dde)-OH. After assembly of the amino acid sequence of the protected RFX-V2a polypeptide chain was complete, the Dde group was removed by treatment with $2 \%$ hydrazine in DMF, then Fmoc-D-Pra-NH-PEG2-COOH was coupled to the side chain amine of $\mathrm{Lys}^{7}$, and that Fmoc group and the N-terminal Fmoc group were removed by treatment with DMF containing $20 \%$ piperidine. The peptidyl-resin was washed with DMF (5 times), $\mathrm{MeOH}$ (2 times), DCM (2 times) and $\mathrm{MeOH}$ (2 times), then dried under vacuum overnight.

Step 5: Cleavage and Deprotection of Alkynyl-PEG2-D-RFX-V2a. Cleavage solution (TFA/TIS/ $\mathrm{H}_{2} \mathrm{O}$ 95/2.5/2.5v/v, $10 \mathrm{~mL} / \mathrm{g}$ peptide Resin) was added into the alkynyl-PEG2-D-RFXV1a resin. The mixture was shaken for $3 \mathrm{~h}$ and the filtrate was collected. Cold ether was added to the filtrate to precipitate the peptide which was collected by centrifugation. The white precipitate was washed with ether twice and dried under vacuum overnight to give crude alkynyl-PEG2-D-RFX-V2a as a white solid. 
Step 6: Purification. Purification of crude alkynyl-PEG2-D-RFX-V2a was performed on a CXTH LC6000/Hanbon NU3000 prep system on YMC C4 silica with columns of dimension $21.2 \times 250 \mathrm{~mm}$. Crude peptides were loaded onto the prep column and eluted at a flow rate of 10 $\mathrm{mL}$ per minute with a shallow gradient of increasing concentrations of solvent $\mathrm{B}(0.1 \%$ TFA in $80 \%$ acetonitrile in water) in solvent A ( $0.1 \%$ TFA in water). Fractions containing the pure target peptide were identified by analytical LC-MS, combined, and lyophilized to give purified alkynyl-PEG2-D-RFX-V2a used for the click reaction with azido-PEG3-D-RFX-V1a.

Step 7: Click Reaction and Purification. Azido-PEG3-D-RFX-V1a and the alkynyl-PEG2D-RFX-V2a were dissolved in ethanol: $\mathrm{H}_{2} \mathrm{O}(\mathrm{v} / \mathrm{v}, 1: 1)$, then $0.2 \mathrm{M} \mathrm{CuSO}_{4}$ was added to the reaction mixture, followed by the addition of $0.2 \mathrm{M}$ of sodium ascorbate, and the reaction mixture was stirred at $30^{\circ} \mathrm{C}$ for $2 \mathrm{~h}$. The reaction mixture was loaded onto RP-HPLC without further workup and purified by gradient elution as described above. Fractions containing the desired product were identified by LC-MS, combined, and lyophilized. (Fig. S5B)

\section{LC-MS analysis of D-proteins}

Analytical RP-HPLC was performed on a HP 1090 system with Waters C4/Phenomenex C18 silica columns $(4.6 \times 150 \mathrm{~mm}, 3.5 \mu \mathrm{m} / 4.6 \times 150 \mathrm{~mm}, 5.0 \mu \mathrm{m}$ particle size $)$ at a flow rate of $1.0 \mathrm{~mL} / \mathrm{min}\left(50^{\circ} \mathrm{C}\right.$ column temperature). Peptides were eluted from the column using a $1.0 \%$ $\mathrm{B} / \mathrm{min}$ gradient of water/0.1\% TFA (solvent $\mathrm{A}$ ) versus $80 \%$ acetonitrile in water/0.1\% TFA (solvent B). Peptide masses were obtained by in-line electrospray MS detection using an Agilent 6120 LC/MSD ion trap. 


\section{Surface plasmon resonance affinity measurements}

Surface plasmon resonance (SPR) binding measurements were carried out on a Biacore S200 (GE). Biotinylated VEGF-A(8-109) was immobilized on a biotin CAPture chip (GE) and serial dilutions of D-proteins were flowed over the chip at $30 \mu \mathrm{L} / \mathrm{min}$ in running buffer $(10 \mathrm{mM}$ Hepes, $\mathrm{pH} 7.4,150 \mathrm{mM} \mathrm{NaCl}, 0.05 \% \mathrm{P} 20)$. Association reactions were 60 seconds for RFX-V1, -V2, V1a and -V2a and 120 seconds for RFX-V1a2a. Dissociation reactions were carried out in running buffer for either 120 seconds (RFX-V1, -V2, -V1a, -V2a) or 360 seconds (RFX-V1a2a). All measurements were carried out at $25{ }^{\circ} \mathrm{C}$. SPR data are representative of multiple independent titrations. Kinetic fits were performed using Biacore software using a global single site binding model.

\section{Expression and purification of VEGF-A for crystallography}

The gene sequence for the VEGF-A (8-109) polypeptide chain was cloned into the expression vector pET21b with a His6-tag and TEV cleavage site sequence added at the Nterminus. The recombinant plasmid was transformed into E. coli BL21-Gold, grown in LB medium supplemented with Ampicillin $(100 \mu \mathrm{g} / \mathrm{ml})$ and expression of the His-tagged protein was induced by $0.3 \mathrm{mM}$ isopropyl- $\beta$-D-thiogalactoside (IPTG) at $16{ }^{\circ} \mathrm{C}$ overnight. Cells were harvested by centrifugation and then stored at $-80^{\circ} \mathrm{C}$.

Pelleted cells from $30 \mathrm{~L}$ of culture were resuspended in $1 \mathrm{~L}$ buffer A $(20 \mathrm{mM}$ Tris, $\mathrm{pH}$ 8.0, $400 \mathrm{mM} \mathrm{NaCl}$ ) and then passed through high-pressure homogenization (3 cycles). His-tagged protein from supernatant was captured on a Ni-NTA resin column $(30 \mathrm{ml})$. The column was washed with $20 \mathrm{CV}$ of Buffer A containing $20 \mathrm{mM}$ imidazole, $5 \mathrm{CV}$ of Buffer C (20mM Tris, pH 8.0, $1 \mathrm{M} \mathrm{NaCl}$ ) and $10 \mathrm{CV}$ of buffer A containing $50 \mathrm{mM}$ imidazole. The His6-tagged-TEV site- 
VEGF-A protein was eluted with a high concentration of imidazole $(0.25 \mathrm{M})$ in buffer A $(5 \mathrm{CV})$.

The eluted protein was digested with TEV protease at a 1:20 ratio (TEV: Protein) and dialyzed against $5 \mathrm{~L}$ buffer (20mM Tris, $\mathrm{pH} 8.0,50 \mathrm{mM} \mathrm{NaCl}$.) at $4{ }^{\circ} \mathrm{C}$ overnight. Cleaved sample was loaded onto a $2^{\text {nd }} \mathrm{Ni}$-NTA column to remove free His-tag. Eluted VEGF-A protein was further purified by ion exchange chromatography on a Resource Q column $(6 \mathrm{ml})$. A final SEC polishing step was performed using HiLoad 16/60 Superdex 75 pg column equilibrated with buffer A. Monodisperse VEGF-A peak fractions were identified by absorbance at $280 \mathrm{~nm}$ and were combined and concentrated to $10-15 \mathrm{mg} / \mathrm{mL}$ in the buffer A. Final purified VEGF-A(8-109) protein was $95 \%$ pure as assessed by SDS-PAGE analysis and the molecular weight was confirmed by direct injection MS.

\section{Crystallography of VEGF-A/D-protein complexes}

$V E G F-A / R F X-V 1$ complex. Crystals for VEGF-A/RFX-V1 were grown by hanging drop vapor diffusion at $18{ }^{\circ} \mathrm{C}$. The drop was composed of $0.8 \mu \mathrm{L}$ of VEGF-A/D-protein complex (2.72 $\mathrm{mg} / \mathrm{ml}$ VEGF-A and $0.5 \mathrm{mM}$ RFX-V1) mixed $1: 1$ with $0.8 \mu$ l of the crystallization solution containing 0.2 M Calcium Chloride, 0.1 M Tris pH 8.5, $18 \%$ w/v PEG 4000. Crystals were soaked in a cryo-protectant solution containing crystallization solution plus $20 \%$ (v/v) glycerol and were flash-frozen in liquid nitrogen. The diffraction data were collected at the Shanghai Synchrotron Radiation Facility beam line BL19U1 to 2.31 Angstroms resolution and processed in space group P2 2121 using XDS. The structure was solved by molecular replacement using Phaser with VEGF structure (PDB ID: 3QTK) as the search model. Structure refinement and model building on the initial model were iteratively performed between Refmac5 and Coot. 
There are two copies of the $\{$ VEGF-A plus RFX-V1\} complex in an asymmetric unit. The detailed data processing and structure refinement statistics are listed in Table S3.

VEGF-A/RFX-V2 complex.

Crystals for VEGF-A/RFX-V2 were grown by hanging drop vapor diffusion at $18{ }^{\circ} \mathrm{C}$. The drop was composed of $0.8 \mu \mathrm{L}$ of VEGF-A/D-protein complex (5.44 mg/ml VEGF-A and 0.46 mM RFX-V2) mixed 1:1 with $0.8 \mu 1$ of the crystallization solution containing $0.15 \mathrm{M}$ Magnesium Chloride, 0.1 M Bis-Tris pH 5.5, 25 \% w/v PEG 3350. Crystals were soaked in a cryo-protectant solution containing crystallization solution plus $10 \%(\mathrm{v} / \mathrm{v})$ glycerol and were flash-frozen in liquid nitrogen. The diffraction data were collected at ALS beam line 8.3.1 to 2.9 Angstroms resolution and indexed in space group $\mathrm{P} 22_{2} 2_{1} 2_{1}$ using XDS. The structure was solved by molecular replacement using Phaser with VEGF structure (PDB ID: 3QTK) as the search model. Structure refinement and model building on the initial model were iteratively performed between Refmac5 and Coot. There are four copies of the \{VEGF-A plus RFX-V2\} complexes in an asymmetric unit. The detailed data processing and structure refinement statistics are listed in Table S3.

All structural images were rendered using Pymol (Schrodinger).

\section{VEGF-A121/VEGFR1-Fc Binding ELISAs}

Biotinylated human VEGF-A121 (isoform 121) was purchased from Acro Biosystems (cat\# VE1-H82E7). VEGFR-1-Fc was purchased from R\&D Systems (cat\# 3516-FL-050). Bevacizumab was manufactured by Genentech Inc. (Lot\# 3067997). In all cases, $1 \mu \mathrm{g} / \mathrm{mL}$ of VEGFR1-Fc was coated on MaxiSorp plates overnight at $4{ }^{\circ} \mathrm{C}$. The following day, coated wells 
were blocked with Super Block (Rockland) for $2 \mathrm{hr}$ with shaking at room temp. For nonequilibrium ELISAs, titrations of D-proteins and bevacizumab were incubated with $1.0 \mathrm{nM}$ of biotinylated VEGF-A121 for 30 min before addition to blocked VEGFR1-Fc coated wells. Antagonist/VEGF-A121 mixture was incubated on VEGFR1-Fc wells for $1 \mathrm{hr}$ with shaking at room temp, washed 3 times with wash buffer (PBS, 0.05\% Tween 20), and bound biotinylated VEGF-A121 was detected with streptavidin-HRP (ThermoFisher). For equilibrium binding ELISAs, titrations of D-proteins, bevacizumab, and soluble VEGFR1-Fc were incubated with $0.15 \mathrm{nM}$ of biotinylated VEGF-A121 overnight at $4{ }^{\circ} \mathrm{C}$ before addition to blocked VEGFR1-Fc coated wells. Antagonist/VEGF-A121 mixture was incubated on VEGFR1-Fc wells for $5 \mathrm{hr}$ with shaking at room temp and developed as above. Data plotted are mean \pm standard deviation of triplicate measurements. $\mathrm{IC}_{50}$ values were derived from 3-parameter fits using Prism (GraphPad) and the error reported is derived from fits.

\section{VEGF cell signaling assay}

Measurement of VEGF cellular signaling was performed using the VEGF Bioassay (Promega). Briefly, HEK293 cells are engineered to express VEGFR-2 coupled to a luciferase response element (KDR/NFAT-RE HEK293). VEGF signaling through VEGFR-2 mediates expression of luciferase which can be quantified using bioluminescence. Plated cells are incubated in the presence of $0.15 \mathrm{nM}$ VEGF-A165 plus D-protein or bevacizumab titrations and incubated at $37^{\circ} \mathrm{C}, 5 \% \mathrm{CO}_{2}$ for 6 hours. Following incubation Bio-Glo is added to wells according to the manufacturer's protocol and relative luminescence units (RLUs) were measured on a PerkinElmer 2300 Enspire Multimode plate reader. Data plotted are mean \pm standard 
deviation of triplicate measurements. $\mathrm{IC}_{50}$ values were derived from 3-parameter fits using Prism (GraphPad) and error reported are derived from fits.

\section{Rabbit wet AMD model}

Dutch Belted rabbits $(1.5-2.5 \mathrm{~kg})$ were purchased from Western Oregon Rabbit Company. aflibercept was purchased from Regeneron Pharmaceuticals. On Day 0 Rabbits were randomized into treatment or control (no drug treatment) groups ( $\mathrm{N}=5$ per group) and baseline ophthalmic exams were done prior to a single intravitreal injection ( $25 \mu \mathrm{L}$ per eye) of RFX-V1a2a (1.0 mg) or aflibercept $(1.0 \mathrm{mg})$. Rabbits were challenged with $1 \mu \mathrm{g}$ VEGF-A 165 in both eyes on Days 2 and 23. On Days 5 and 26 fluorescein angiography was performed on both eyes and images were taken to assess vascular leakage. Scoring of vascular leakage based on FA images was carried out at Day 5 and 26. Overall score for each eye was calculated as the average of the 3 O'clock and 9 O'clock score and plotted in Figure 5B. All the procedures related to animal handling, care and the treatment in the study were performed according to the guidelines approved by the Institutional Animal Care and Use Committee (IACUC) of Absorption Systems LLC following the guidance of the Association for Assessment and Accreditation of Laboratory Animal Care (AAALAC).

MC38 syngeneic tumor model in C57BL6 mice

Female C57BL6 mice transgenic for human PD-1 (12-13 weeks) were purchased from Beijing Biocytogen Co. Nivolumab was purchased from Bristol Myers Squibb, lot \#AAY1999. MC38 tumor cells $\left(1 \times 10^{6}\right)$ were implanted subcutaneously in the right front flank and tumors were allowed to establish until the mean volume was $82 \pm 8 \mathrm{~mm}^{3}$. Mice were randomized into 
treatment groups $(\mathrm{N}=6$ per group) on Day 0 when treatment initiation began. RFX-V1a2a at 2 $\mathrm{mg} / \mathrm{kg}$ or $6 \mathrm{mg} / \mathrm{kg}$ was injected i.p. daily for 2 weeks (14 doses) and nivolumab at $1 \mathrm{mg} / \mathrm{kg}$ or 3 $\mathrm{mg} / \mathrm{kg}$ was injected i.p. biweekly for 6 doses. Serum samples were taken from terminal bleeds at Day 22 and assayed for anti-drug antibodies using a murine IgG ELISA. Briefly, equimolar concentrations of nivolumab and RFX-V1a2a were titrated and coated on MaxiSorp plates overnight at $4{ }^{\circ} \mathrm{C}$ in PBS. The following day, plates were blocked with Super Block (Rockland) and diluted serum from each mouse (1:800 in PBS + 0.05\% tween $20+0.5 \%$ BSA) was incubated on the coated antigens, washed, and bound mouse IgG was detected using 1:2000 diluted anti-mu-Fc-HRP (MilliporeSigma, cat\#12349). All the procedures related to animal handling, care and the treatment in the study were performed according to the guidelines approved by the Institutional Animal Care and Use Committee (IACUC) of Shanghai SIPPR-BK Laboratory Animal Co., Ltd following the guidance of the Association for Assessment and Accreditation of Laboratory Animal Care (AAALAC).

\section{Subcutaneous immunization in BALB/c mice}

Titermax adjuvant was purchased from TiterMax, Inc. Bevacizumab was purchased from Genentech/Roche. Female BALB/c mice (6-8 weeks) were randomized into immunization groups on Day 0 ( $\mathrm{n}=5$ per group). Immunizations were performed on Days $0,21,35$ by subcutaneous injection of $25 \mu \mathrm{g}$ of antigen. Antigens were emulsified in water-oil microparticle adjuvant (TiterMax) for injection on Day 0 and administered in PBS for Days 21 and 35. Serum pre-bleeds were performed on Days 0,21, 35 prior to immunizations. Final bleeds for max titer response were taken on Day 42. Serum samples were assayed for anti-drug antibodies using a murine IgG ELISA. Briefly, equimolar concentrations of nivolumab, bevacizumab, and RFX- 
V1a2a were titrated and coated on MaxiSorp plates overnight at $4{ }^{\circ} \mathrm{C}$ in PBS. The following day, plates were blocked with Super Block (Rockland) and diluted serum from each mouse (1:800 in PBS $+0.05 \%$ tween $20+0.5 \%$ BSA) was incubated on the coated antigens, washed, and bound mouse IgG was detected using 1:2000 diluted anti-mu-Fc-HRP (MilliporeSigma, cat\#12349). All the procedures related to animal handling, care and treatment in the study were performed according to the guidelines set forth in an ACUP protocol for polyclonal antisera production in mice, approved by the Institutional Animal Care and Use Committee (IACUC) of Josman LLC. 
A

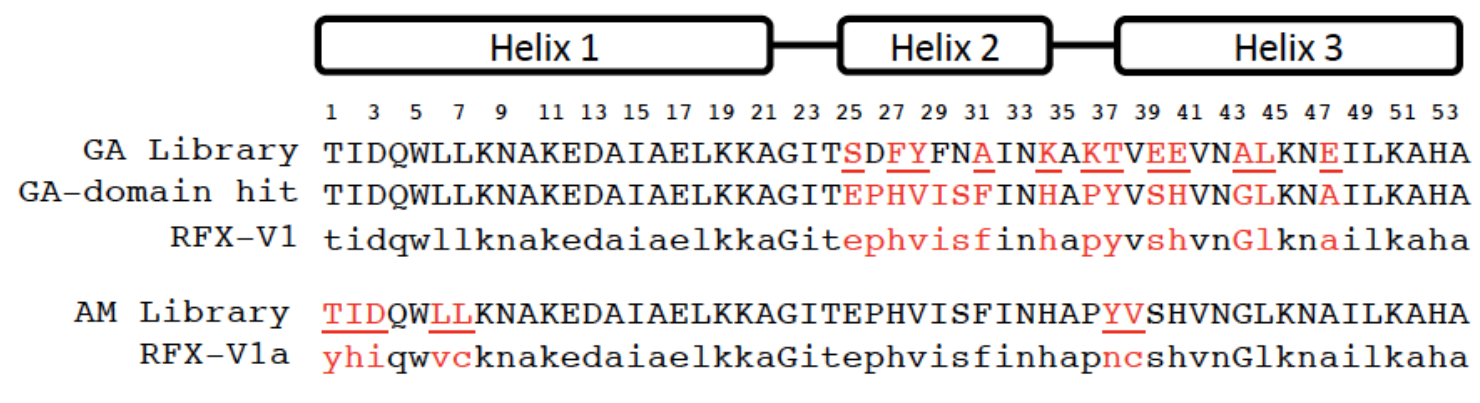

B

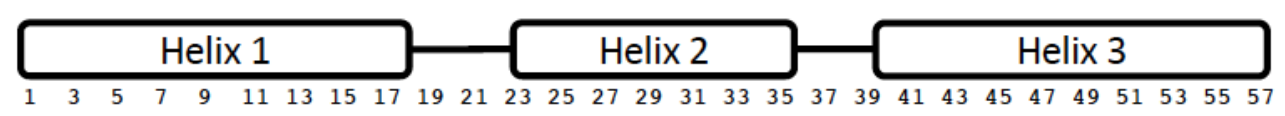

Z Library VDNKFNKEQQNAFYEILHLPNLNEEQRNAFIOSLKDDPSQSANLLAEAKKLNDAQAPK

Z-domain hit VDNKFNKEWDNAWREIRHLPNLNLEQKRAFISSLYDDPSQSANLLAEAKKLNDAQAPK

RFX-V2 vdnkfnkewdnawreirhlpnlnleqkrafisslyddpsqsanllaeakklndaqapk

AM Library VDNKFNKEWDNAWㅌEIRHLPNLNLEQKRAFISSSLYDDPSQSANLLAEAKKLNDAQAPK

RFX-V2a vdnkfnkewdnawreirhlpnlnveqkr̄afir̄slyddpsqsanllaeakklndaqapk

C

RFX-V1a2a [yhiqwvcknakedaiael-k(Azidoacety1-PEG3)-kaGitephvisfinhapncs hvnGlknailkaha-NH2 ]-triazole-[vdnkfn-k (D-Pra-PEG2) ewdnawreirhlpnlnveqkrafirslyddpsqsanllaeakklndaqapk-NH2 ]

Figure S1. Sequences of phage display libraries and proteins. Amino acid sequences are given in the single letter code; uppercase letters are L-amino acids and glycine; lower case letters are Damino acids. (A) GA-domain scaffold sequence and library used for panning. Underlined residues in GA library were randomized with NNK codons for full amino acid diversity. Underlined residues in the AM library were randomized with NNC codons for 15 amino acid diversity including cysteine. (B) Z-domain scaffold sequence and library used for panning. Underlined residues in GA library were randomized using trinucleotide codons for full amino acid diversity excluding cysteine. Underlined residues in the AM library were soft-randomized using codons to incorporate $30 \%$ mutation rate at each amino acid. (C) Amino acid sequence and covalent structure of the heterodimeric D-protein RFX-V1a2a. 


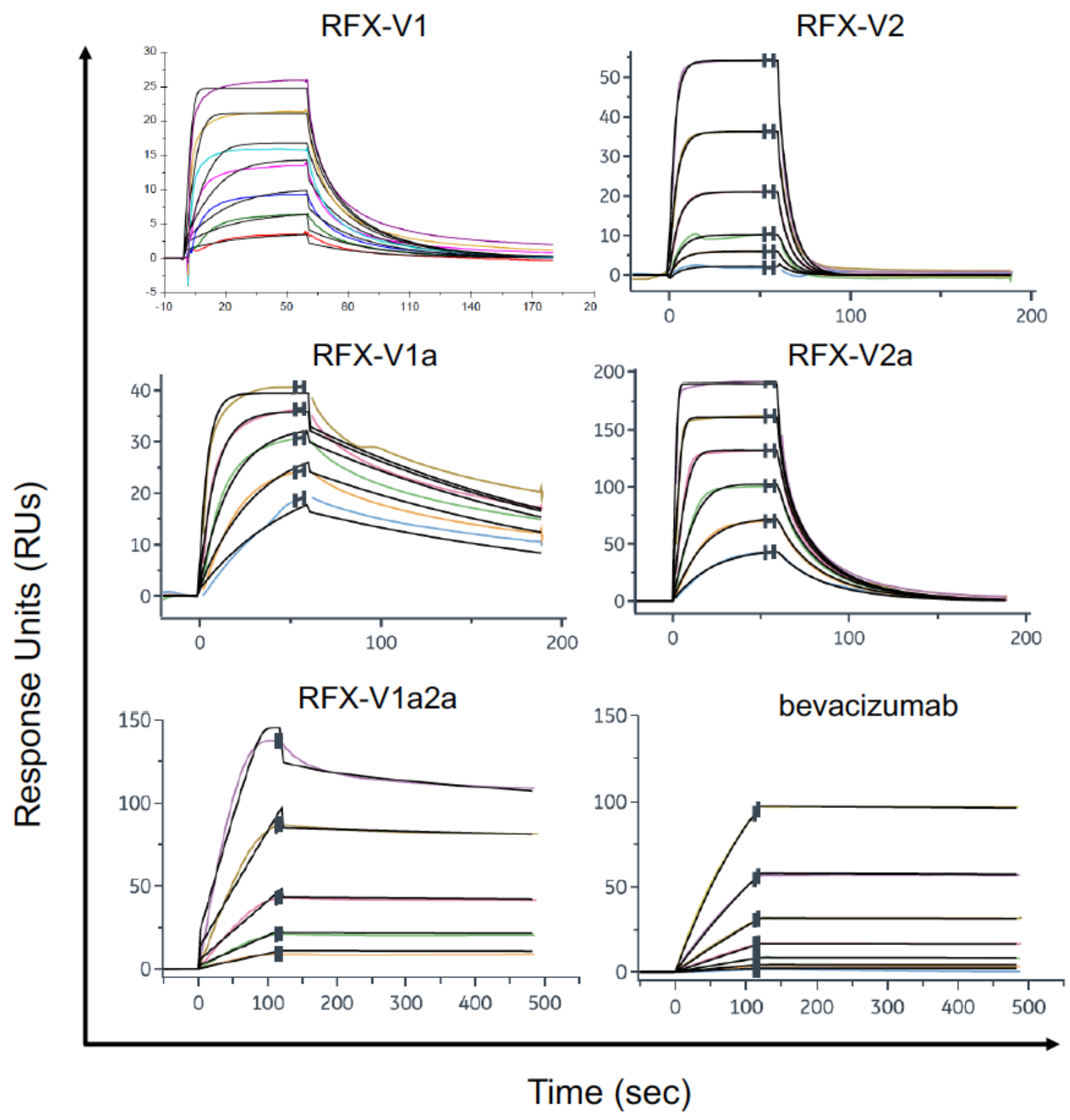

Figure S2. SPR sensorgrams of kinetic binding parameters measured for D-proteins and bevacizumab. 


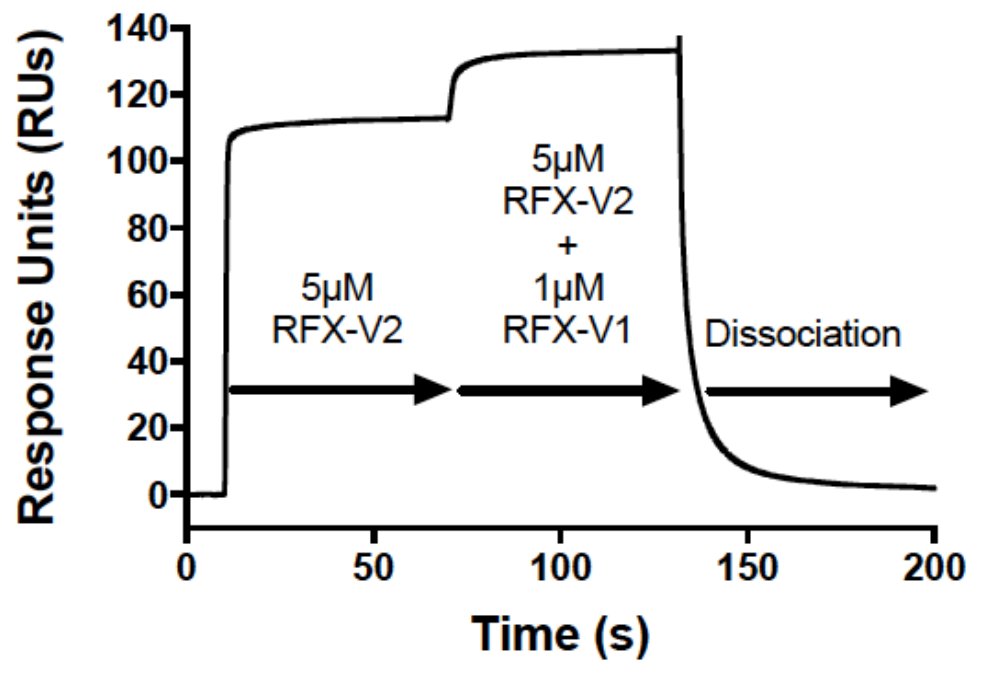

Figure S3. SPR-based epitope binning of RFX-V2 and RFX-V1. In the first association step, 5 $\mu \mathrm{M}$ of RFX-V2 is used to saturate VEGF-A on the chip surface. In the second association step, $1 \mu \mathrm{M}$ of RFX-V1 is included with $5 \mu \mathrm{M}$ of RFX-V2 and exhibits additive binding to VEGF-A indicating the site for RFX-V1 is not blocked by RFX-V2. Both D-proteins display complete dissociation from VEGF-A. 

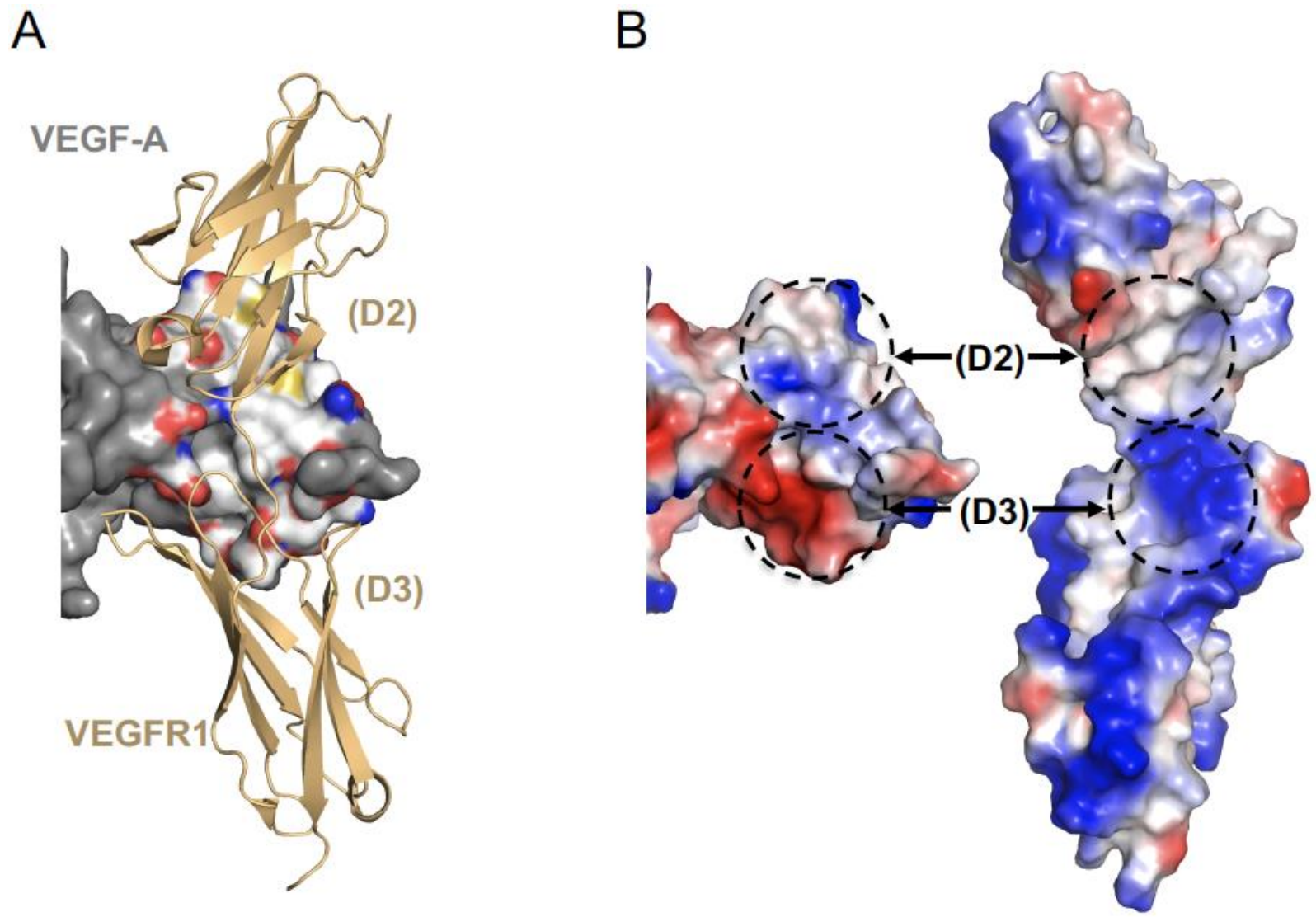

Figure S4. Structural characterization of the VEGF-A/VEGFR-1 contacts. (A) Previous structure solved for VEGF-A (grey) in complex with VEGFR-1 (light orange) depicting the epitope on VEGF-A contacted by D2 and D3 Ig-domains of VEGFR-1 colored by element (white carbon, red oxygen, blue nitrogen, and yellow sulfur) (PDB ID: 5T89, 24). (B) Open book representation of (A) with the D2 and D3 domains rotated 180 degrees away from VEGF-A and electrostatic surface potential shown for both molecules. The D2 and D3 binding sites are encircled highlighting the predominant non-polar hydrophobic nature of the D2 interaction and polar hydrophilic nature of the D3 interaction. 
Fig S5

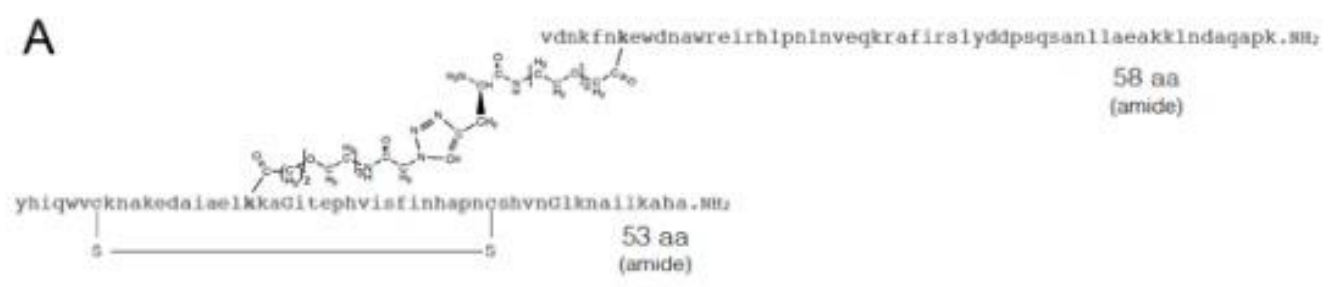

B

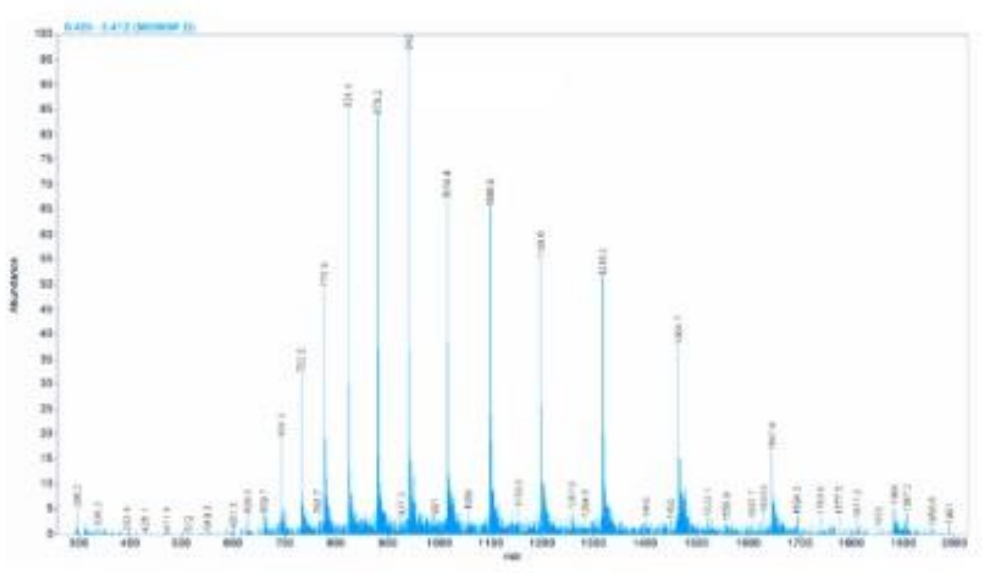

C

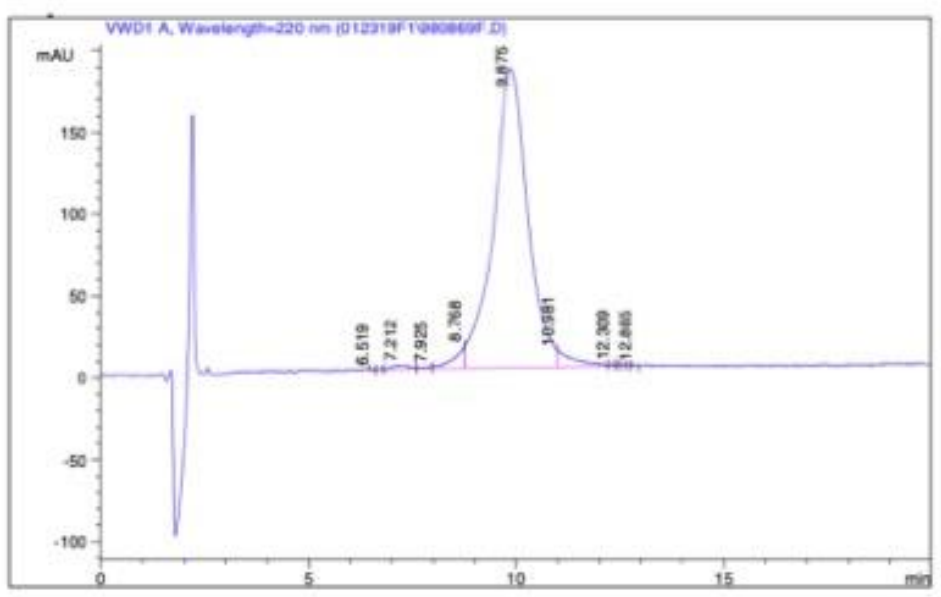

Figure S5. Chemical structure (A), MS spectrum (B), and LC chromatogram (C) for RFX-V1a2a following chemical synthesis and purification. Observed mass (LC-MS): $13174 \pm 1 \mathrm{Da}$; Calculated mass (average isotope composition): $13174.8 \mathrm{Da}$ 
Table S1. Summary of SPR-derived kinetic binding parameters for D-proteins and bevacizumab.

\begin{tabular}{cccc} 
D-protein & $k_{a}\left(\mathrm{M}^{-1} \mathrm{~s}^{-1}\right)$ & $k_{d}\left(\mathrm{~s}^{-1}\right)$ & $K_{D}(\mathrm{nM})$ \\
\hline RFX-V1 & $4.8 \times 10^{6}$ & $2.1 \times 10^{-1}$ & 43 \\
RFX-V2 & $8.9 \times 10^{5}$ & $1.8 \times 10^{-1}$ & 200 \\
RFX-V1a & $2.1 \times 10^{6}$ & $5.2 \times 10^{-3}$ & 2.4 \\
RFX-V2a & $1.2 \times 10^{7}$ & $2.1 \times 10^{-1}$ & 17 \\
RFX-V1a2a & $1.7 \times 10^{7}$ & $1.3 \times 10^{-3}$ & 0.08 \\
bevacizumab & $1.8 \times 10^{5}$ & $3.0 \times 10^{-5}$ & 0.16 \\
\hline
\end{tabular}


Table S2. Summary of IC 50 values for D-proteins and bevacizumab blocking VEGF-A121 binding to VEGFR1-Fc in non-equilibrium ELISA.

\begin{tabular}{cc} 
D-protein & $\begin{array}{c}\text { VEGF-A121 } \\
\text { Non-equilibrium } \\
\text { Blocking ELISA } \\
\text { IC50 }(\mathrm{nM})\end{array}$ \\
\hline RFX-V1 & $52 \pm 12$ \\
RFX-V2 & $940 \pm 580$ \\
RFX-V1a & $3.5 \pm 0.6$ \\
RFX-V2a & $1700 \pm 1300$ \\
bevacizumab & $1.8 \pm 0.3$ \\
\hline
\end{tabular}


Table S3. Data collection and refinement statistics for VEGF/ D-protein complexes

\begin{tabular}{|c|c|c|}
\hline & VEGF-A/RFX-V1 & VEGF-A/RFX-V2 \\
\hline \multicolumn{3}{|l|}{ Data Collection } \\
\hline Beam line & SSRF BL19U1 & ALS 8.3.1 \\
\hline Space group & $\mathrm{P} 2{ }_{1} 2_{1} 2_{1}$ & $\mathrm{P} 2{ }_{1} 2_{1} 2_{1}$ \\
\hline Wavelength $(\AA)$ & 0.97 & 1.11 \\
\hline \multicolumn{3}{|l|}{ Cell dimensions } \\
\hline$a, b, c(\AA)$ & $61.9,69.1,89.4$ & $66.8,120.4,120.4$ \\
\hline$\left.\alpha, \beta, \chi^{\circ}\right)$ & $90,90,90$ & $90,90,90$ \\
\hline Mol/asymmetric unit & 2 & 4 \\
\hline Mol/unit cell & 8 & 16 \\
\hline Resolution $(\AA)$ & $46.09-2.31(2.39-2.31)$ & $120.4-2.9(3.08-2.90)$ \\
\hline$R_{\text {merge }}{ }^{\mathrm{a}}$ & $0.044(0.241)$ & $0.059(1.055)$ \\
\hline$I / \sigma I$ & $25.9(7.0)$ & $25.7(2.4)$ \\
\hline Completeness (\%) & $99.9(100.0)$ & $99.9(99.9)$ \\
\hline Redundancy & $6.4(6.5)$ & $12.8(12.7)$ \\
\hline \multicolumn{3}{|l|}{ Refinement } \\
\hline Resolution & $54.67-2.31(2.37-2.31)$ & $58.45-2.90(2.98-2.90)$ \\
\hline No. of reflections & $16353(1178)$ & $21152(1484)$ \\
\hline$R_{\text {work }}(\%) / R_{\text {free }}(\%)$ & $26.0 / 31.4$ & $26.3 / 31.5$ \\
\hline \multicolumn{3}{|l|}{ No. of atoms } \\
\hline Non-solvent & 2430 & 4936 \\
\hline Solvent & 60 & 0 \\
\hline Average B-factor $\left(\AA^{2}\right)$ & 43.6 & 126.9 \\
\hline \multicolumn{3}{|l|}{ R.M.S deviations } \\
\hline Bond lengths $(\AA)$ & 0.01 & 0.01 \\
\hline Bond angles $\left({ }^{\circ}\right)$ & 1.24 & 1.80 \\
\hline
\end{tabular}

${ }^{\mathrm{a}} R_{\text {merge }}=\Sigma \mid \mathrm{I}-\langle\mathrm{I}>| / \sum \mathrm{I} .{ }^{\mathrm{b}} R_{\text {free }}$ calculated using $5 \%$ of the data 
Table S4. Summary of IC50 values for D-proteins and bevacizumab blocking VEGF-121A binding to VEGFR1-Fc in an equilibrium binding ELISA and VEGF-A signaling inhibition in a cell signaling assay.

\begin{tabular}{ccc}
$D$-protein & VEGF-A121 & \\
& Equilibrium & VEGF-A Signaling \\
& Blocking ELISA & Assay \\
& $I C_{50}(\mathrm{nM})$ & $I C_{50}(\mathrm{nM})$ \\
\hline RFX-V1a & $7.6 \pm 1.3$ & $6.1 \pm 1.2$ \\
RFX-V1a2a & $0.31 \pm 0.06$ & $0.39 \pm 0.02$ \\
bevacizumab & $0.70 \pm 0.20$ & $0.53 \pm 0.10$ \\
VEGFR1-Fc & $0.23 \pm 0.04$ & - \\
\hline
\end{tabular}

\section{Reference:}

1. Mandal, K., and Kent, S. B. H. (2011) Total chemical synthesis of biologically active vascular endothelial growth factor. Angew. Chem., Int. Ed. 50, 8029-8033.

2. Mandal, K., Uppalapati, M., Ault-Riche, D., Kenney, J., Lowitz, J., Sidhu, S. S., and Kent, S. B. H. (2012) Chemical synthesis and X-ray structure of a heterochiral \{D-protein antagonist plus vascular endothelial growth factor $\}$ protein complex by racemic crystallography. Proc. Natl. Acad. Sci. U. S. A. 109, 14779-14784.

3. Fang, G.-M., Li, Y.-M., Shen, F., Huang, Y.-C., Li, J.-B., Lin, Y., Cui, H.-K., and Liu, L. (2011) Protein chemical synthesis by ligation of peptide hydrazides. Angew. Chem., Int. Ed. $50,7645-7649$.

4. Sidhu, S. S., Feld, B. K., and Weiss, G. A. (2006) M13 Bacteriophage Coat Proteins Engineered for Improved Phage Display. Protein Eng. Protoc. 352, 205-220.

5. Kunkel, T. A. (1985) Rapid and efficient site-specific mutagenesis without phenotypic selection. Proc. Natl. Acad. Sci. U. S. A. 82, 488-492. 